\title{
On the use of circulating desphospho-uncarboxylated matrix gla-protein to determine symptomatic atherosclerotic calcification phenotype
}

\author{
Rachel Cahalane $^{1}$, Hilary Barrett ${ }^{1}$, Aisling Ross ${ }^{1}$, John Mulvihill ${ }^{1}$, Helen Purtill ${ }^{2}$, \\ Eamon Kavanagh ${ }^{3}$, Tony Moloney ${ }^{3}$, Julie O'Brien ${ }^{4}$, Cees Vermeer ${ }^{5}$, Paula O'Shea', \\ Siobhan Egan', Fiona Leahy ${ }^{7}$, Michael Walsh ${ }^{1}$ and Eibhlis O’Connor ${ }^{8,9}$ \\ ${ }^{1}$ Bernal Institute, School of Engineering, University of Limerick, Limerick, Ireland, \\ ${ }^{2}$ Department of Mathematics and Statistics, University of Limerick, Limerick, Ireland, \\ ${ }^{3}$ Department of Vascular Surgery, University Hospital Limerick, Limerick, Ireland, \\ ${ }^{4}$ Department of Radiology, University Hospital Limerick, Limerick, Ireland, \\ ${ }^{5} R \& D$ Group, VitaK, Maastricht, Netherlands, \\ ${ }^{6}$ Biochemistry Lab, University Hospital Galway, Galway, Ireland, \\ ${ }^{7}$ Clinical Research Support Unit, University Hospital Limerick, Limerick, Ireland, \\ ${ }^{8}$ School of Natural Sciences and Department of Biological Sciences, University of Limerick, Limerick, Ireland and \\ ${ }^{9}$ Alimentary Pharmabiotic Centre, Microbiome Institute, University College Cork, Cork, Ireland
}

\section{Abstract}

The rupture of atherosclerotic plaques is the prerequisite for adverse cardiovascular events. Calcification morphology plays a critical role in plaque stability, therefore accurate calcification classification is essential for favourable patient management. Blood biomarkers may be a worthwhile approach to stratify patients based on calcification phenotype. Vitamin K-dependent Matrix $\gamma$-carboxyglutamate (Gla) protein (MGP) is a potent inhibitor of vascular calcification. Recent studies have demonstrated the potential utility of circulating non-functional MGP (dp-ucMGP) measurements to determine arterial stiffness and calcification levels. The objective of this study was to examine the relationship between circulating dp-ucMGP and calcification phenotype within symptomatic atherosclerotic lesions. Consenting patients undergoing standard endarterectomy procedures were recruited $(\mathrm{n}=29)$. Fasting venous blood was collected preoperatively. Circulating plasma levels of dp-ucMGP were quantified using the inaKtif MGP (dp-ucMGP) iSYS kit. A bicinchoninic acid assay was used to standardise the total protein content present in each sample. High-resolution micro-CT imaging was conducted on the excised atherosclerotic specimens postoperatively. ImageJ post-processing was used to accurately quantify the calcification volume ( $\geq 130$ Hounsfield Units) and determine the total number of calcified particles (3D objects counter plugin). Thirteen carotid (average age 71 years, 9 male) and fourteen peripheral lower limb (average age 65 years, 12 male) patients were examined. One patient had a carotid and a peripheral lower limb plaque (age 79, male). Peripheral lower limb specimens have larger volumes of calcification and higher numbers of calcified particles than carotid samples $(472 \pm 310$ vs $85 \pm$ $113 \mathrm{~mm}^{3}, \mathrm{p}<0.0005 ; 13919 \pm 16034$ vs $3476 \pm 6208, \mathrm{p}=0.061$.) While a higher dp-ucMGP value was noted in carotid than peripheral lower limb patients $(214 \pm 52$ vs $169 \pm 36 \mathrm{pmol} / \mathrm{L}, \mathrm{p}=0.014)$ there was no correlation between circulating dp-ucMGP and calcification volume or number of calcified particles $\left(r_{s}=-0.329\right.$ and $\left.r_{s}=0.046\right)$. Previous research also found that peripheral lower limb lesions contain higher volumes of calcification than carotid lesions. There is currently no published data on calcified particle comparisons. Patients with symptomatic carotid disease are assumed to have a degree of peripheral arterial disease, this could explain the higher levels of circulating dp-ucMGP in carotid patients. The current study did not examine the dietary patterns of individuals with regards to Vitamin $\mathrm{K}$ intake or analyse other areas of the vasculature for additional calcification. This may interfere with dp-ucMGP measurements. This study serves as a preliminary investigation into the potential of dp-ucMGP as a blood based biomarker to distinguish between symptomatic atherosclerotic calcification phenotypes.

\section{Conflict of Interest}

There is no conflict of interest 